\title{
AVALIAÇÃO DE DENSIDADES PARA A PREVISÃO DOS RETORNOS DAS AÇÕES DA PETROBRAS
}

\author{
André de Mattos Marques ${ }^{1}$
}

RESUMO: A emissão primária de ações pode ser uma importante fonte de financiamento para a criação de capacidade produtiva, em particular, em um ambiente de oligopólio com limitado crédito bancário. Além de favorecer a acumulação de capital da empresa, com baixos custos monetários, permite a redução do endividamento empresarial (de alto custo) junto ao setor bancário. No contexto das descobertas de petróleo na camada pré-sal da Bacia de Santos, dos desafios tecnológicos e da necessidade de novos investimentos daí derivados, muitas emissões primárias foram realizadas pela Petrobras e o mercado apresentou grande volatilidade e imprevisibilidade na última década. O objetivo do artigo foi testar três densidades de probabilidade para prever as probabilidades dos retornos das ações (PETR4) da Petrobras negociadas na BOVESPA a partir dos preços diários de fechamento no período de 26.06.2000 a 17.04.2012. As densidades candidatas foram a Normal, a Logística e a Cauchy. O teste de aderência de Kolmogorov-Smirnov sugere que a densidade Logística é a que melhor descreve a probabilidade dos retornos das ações da Petrobras. Os resultados indicam que o investidor pode obter uma rentabilidade diária positiva com probabilidade de 0,5130271 e uma rentabilidade maior do que $5 \%$ ao mês com uma probabilidade de 0,4698542 .

Palavras-chave: retorno das ações; ajuste; densidade de probabilidade.

ABSTRACT: The primary issue of shares may be an important source of funding for the creation of productive capacity, particularly in an oligopolistic environment with limited bank credit. In addition to favoring the accumulation of capital of the company, with low monetary costs, it allows the reduction of corporate debt (expensive) with the banking sector. In the context of oil discoveries in the Brazil, and on the technological challenges and the need for new investment in fixed capital derived thereof, many primary issues were made and the stock market showed great volatility and unpredictability in the last decade. The objective was to test three densities of probability to predict the odds of daily stock returns (PETR4) Petrobras traded on BOVESPA, from the daily closing prices for the period 26.06.2000 to 17.04.2012. The candidate density functions were Normal, Logistic and Cauchy. The adherence test of Kolmogorov-Smirnov suggests that the Logistic density is the one that best describes the probability of stock returns of Petrobras. The results indicate that the trader can obtain a profitability daily positive probability 0.5130271 and a yield greater than $5 \%$ per month with a probability of 0.4698542 .

Key words: stock return; density; probability; fit.

\footnotetext{
${ }^{1}$ Professor Adjunto do Departamento de Economia da Universidade Federal do Rio Grande do Norte (UFRN). Email: andremmarques@yahoo.com.br.
}

Recebido em: 12/06/2012

Aceito em: 23/07/2012 


\section{INTRODUÇÃO}

A possibilidade de se obter alta rentabilidade no mercado financeiro com uma probabilidade elevada pode ser um grande atrativo do mercado de ações. Por essa via, os investidores (poupadores) aumentam os recursos financeiros das empresas, favorecem a redução de seu endividamento junto aos bancos comerciais (reduzindo o risco dos empréstimos) e contribuem para a expansão de sua capacidade produtiva, seja pela aquisição de máquinas e equipamentos e/ou incorporação de novas tecnologias.

A importância do financiamento pela emissão de ações pode ser assim sintetizada. Sem as sociedades de capital por ações, toda a poupança proveniente dos lucros representa uma acumulação de recursos empresariais; todas as demais poupanças devem ser obtidas por meio de empréstimos bancários. Com o sistema de capital por ações, as empresas são capazes de obter as poupanças externas (à firma) sem ter que tomá-las por empréstimo, por isso, sem aumentar seu endividamento que é limitado pelo princípio do risco crescente. Isto é, financiamento pela emissão de ações pela emissão primária de ações impede que os empréstimos alcancem certo limite além do qual o risco empresarial não seria tolerável pelos bancos.

Com as novas descobertas de petróleo no Brasil, emergiu a necessidade de crescentes investimentos em capital e tecnologias para exploração e extração de petróleo e gás pela Petrobras. Apesar de o crédito bancário apresentar trajetória de expansão no Brasil, a emissão de ações, em ambiente de oligopólio, ainda é o meio mais barato de obter grandes financiamentos, uma vez que haja grande liquidez no mercado. Neste contexto favorável, a estatal brasileira fez grandes emissões primárias nos últimos anos, aumentando fortemente sua participação na Bolsa de Valores de São Paulo (BOVESPA).

A teoria econômica reconhece que há uma conexão entre o mercado acionário e o mercado monetário (mercado de títulos públicos), à medida que o retorno das ações compete com a taxa real de juros dos títulos (renda fixa). Isto é, à medida que aumenta a expectativa de inflação ou que a autoridade monetária defina uma taxa nominal de juros menor, reduz-se a taxa real de juros, por isso, espera-se um aumento na demanda por ações, o que pode elevar seus preços e também o seu retorno, e vice-versa. Neste sentido, a trajetória de redução da taxa 
básica de juros no Brasil, aliada à ocorrência de um regime de baixa inflação na última década, pode ter contribuído positivamente para o desempenho do mercado acionário no Brasil e para as captações da Petrobras.

Todavia, uma grande variedade de eventos econômicos e financeiros externos, tais como a crise do mercado financeiro norte-americano em 2008 e a atual ameaça de desagregração na área do Euro também podem influenciar o retorno das ações das diversas empresas que constituem o índice IBOVESPA, dentre elas a Petrobras, provocando grande volatilidade e imprevisibilidade em seu comportamento. Em particular, a volatilidade geralmente cresce com a rentabilidade esperada, e a ausência de autocorrelação entre os retornos torna difícil sua previsão a partir de modelos lineares auto-regressivos convencionais.

Neste caso, o cálculo das probabilidades pode prestar um importante serviço. O ajuste de uma função densidade de probabilidade (f.d.p.) pode oferecer indicações mais precisas sobre a rentabilidade esperada da aplicação e sua probabilidade associada, desde que se conheça a densidade de probabilidade que melhor descreve seu comportamento. Desse modo, pode-se efetuar um planejamento mais eficiente dos ganhos futuros a serem obtidos no mercado financeiro. Para realizar essa tarefa, todavia, é necessário verificar a aderência de uma função densidade de probabilidade adequada à série de rentabilidade das ações de interesse.

Em síntese, o problema de se ajustar uma densidade de probabilidade é tal que, se o pesquisador dispõe de uma coleção de observações $x_{1}, x_{2}, \ldots, x_{n}$, e se ele deseja testar se essas observações, que foram extraídas de uma população desconhecida, pertencem a uma população com uma f.d.p. $f(x, \boldsymbol{\theta})$ específica, onde $\boldsymbol{\theta}$ é um vetor de parâmetros a ser estimado a partir das observações disponíveis, então é necessário: (a) formular uma hipótese sobre as possíveis densidades candidatas; (b) estimar os parâmetros das densidades candidatas; (c) analisar a qualidade do ajuste; (d) testar as densidades escolhidas. Ao final, poder-se-á, então, a partir da densidade que melhor descreve o comportamento de $x$, prever as probabilidades da variável de interesse.

Existem muitas distribuições teóricas de probabilidade para variáveis aleatórias discretas e também muitas funções densidade de probabilidade para variáveis aleatórias contínuas. No caso das variáveis aleatórias discretas, dentre as mais conhecidas estão a 
distribuição de Bernoulli, a Binomial, Binomial Negativa, Hipergeométrica, Geométrica e Poisson.

Para o caso das variáveis aleatórias contínuas, dentre as funções densidade mais conhecidas, estão a densidade Uniforme, Normal, Log-Normal, Gama, Valores Extremos (ou Gumbel), Weibull, Exponencial, Beta, Qui-Quadrado, F de Snedecor, Logística e Cauchy. O ajuste de uma f.d.p. para o exercício de simulação e planejamento de atividades tem sido muito comum no campo da climatologia ${ }^{2}$. Essa abordagem pode ser estendida, todavia, para qualquer evento da natureza, posto que seja aleatório.

No geral, os testes de aderência mais empregados em estudos com essa finalidade têm sido o Qui-Quadrado, Lilliefors, Shapiro-Wilk, Cramer-von Mises e Anderson-Darling. O Teste escolhido para indicar o grau de ajuste da f.d.p. neste estudo foi o teste KolmogorovSmirnov (KS), em vista de sua grande versatilidade frente aos demais, uma vez que pode ser empregado para investigar o grau de ajuste de qualquer densidade de probabilidade de interesse do pesquisador.

Os demais testes têm uma aplicabilidade bem mais específica. O teste de aderência Qui-Quadrado, por exemplo, requer que a freqüência de uma classe não seja inferior a cinco e que os dados sejam grupados, com perda de informação. Isso não ocorre com o teste KS, pois os dados não precisam ser grupados, podendo ser testados isoladamente, sem perda de informação. Além disso, por ser um teste não-paramétrico, pode ser aplicado também a pequenas amostras (menos de 30 observações).

O objetivo do trabalho foi testar três densidades de probabilidade para prever o comportamento da rentabilidade diária das ações da Petrobras (PETR4) calculada a partir do preço de fechamento das ações preferenciais negociadas na BOVESPA, para o período de 26/06/2000 a 17/04/2012. A partir das medidas descritivas do retorno das ações e das características da variável, foram selecionadas três densidades candidatas. A estratégia adotada no trabalho obedeceu a quatro passos para o ajuste da função densidade e o cálculo das probabilidades: 1) escolha de funções densidades de probabilidade candidatas, com base nas características da variável observada; 2) estimativa dos parâmetros; 3) aplicação do teste

\footnotetext{
${ }^{2}$ Ver, por exemplo, Cargnelutti Filho et. al. (2004); Murta et. al. (2005).
} 
Kolmogorov-Smirnov; 4) cálculo das probabilidades de rentabilidade a partir da Função de Distribuição Acumulada.

O artigo está organizado do seguinte modo: na seção dois é descrita a base de dados e a metodologia utilizada no trabalho; na seção três são apresentados e discutidos os resultados obtidos; e, na seção quatro são feitos comentários finais.

\section{BASE DE DADOS E METODOLOGIA}

\subsection{Base de dados}

A seleção das ações PTR4 da Petrobras levou em consideração, primeiro, a importância teórica que o financiamento do estoque de capital pela emissão de ações tem para as empresas em geral, em especial, em uma estrutura de oligopólio (Steindl, 1983; Labini, 1986). Ademais, examinando-se a quantidade teórica, dentre todas as 68 empresas que atualmente constituem o índice IBOVESPA, as ações da Petrobras têm a segunda maior participação, com 7,589\% do total de ações que compõem o índice.

A maior participação na quantidade teórica do IBOVESPA refere-se às ações da Vale do Rio Doce, com 9,586\%. A média de participação das demais empresas na quantidade teórica que compõem o IBOVESPA situa-se em 1,25\%. Quando se examina o critério de liquidez, dentre as empresas petrolíferas que lançam ações no IBOVESPA, com os dados da tabela 1, abaixo. Constata-se que são as ações preferenciais da Petrobras (PETR4) que têm a maior liquidez no mercado.

Tabela 1: Índice de liquidez das ações das empresas de petróleo negociadas na BOVESPA

\begin{tabular}{l|l|c}
\hline \multicolumn{1}{c|}{ Ação } & \multicolumn{1}{c|}{ Tipo } & Índice de liquidez \\
\hline EXXON MOBIL & DRN MB & $* * *$ \\
\hline HRT PETROLEO & ON NM & 0,234 \\
\hline OGX PETROLEO & ON NM & 3,840 \\
\hline PET MANGUINH & ON & 0,184 \\
\hline PET MANGUINH & PN & 0,022 \\
\hline PETROBRAS & ON & 2,168 \\
\hline PETROBRAS (PETR4) & PN & $\mathbf{6 , 4 6 1}$ \\
\hline QGEP PART & ON NM & 0,373 \\
\hline \hline
\end{tabular}

Fonte: BOVESPA. (***) informação indisponível. 
A amostra empregada no trabalho compreende 3028 observações, correspondendo à rentabilidade das ações PETROBRAS PN (PETR4), negociadas na BOVESPA nos dias úteis do período de 26 de junho de 2000 a 17 de abril de 2012, disponível na página eletrônica da Economatica (http://www.economatica.com/). O período selecionado refere-se à consolidação do regime de câmbio flexível na economia brasileira, com metas de inflação e superávit primário. O software utilizado para realizar todas as estimativas e cálculos de probabilidade foi o Ambiente $\boldsymbol{R}$.

O comportamento do preço das ações aparece no Gráfico 1, abaixo. Por inspeção visual, constata-se que o comportamento das ações pode estar relacionado com os indicadores mais amplos da economia e com os maiores eventos que afetaram a economia brasileira nesse período, tais como o overshooting cambial das eleições presidenciais de 2002, e especialmente, a crise dos mercados financeiros nos EUA em 2007/2008. Em especial, observa-se que a trajetória de alta acentua-se nos períodos em que houve grandes descobertas de petróleo no Brasil, na Bacia de Santos (camada pré-sal).

Em 11 de julho de 2006 a Petrobras anunciou a descoberta de petróleo em águas ultraprofundas da Bacia de Santos, no estado do Rio de Janeiro. Em setembro de 2007, a empresa divulgou a estimativa do volume de óleo recuperável: 5 a 8 bilhões barris de petróleo e gás natural. Quatro anos depois, em fevereiro de 2010, testes de longa duração e a perfuração de poços confirmaram a expectativa inicial da empresa. Confirmando a expectativa de uma grande jazida petrolífera, inúmeras descobertas foram anunciadas entre 2006 e 2011, que receberam diversas denominações: Franco, Libra, Parati, Carioca, Caramba, Caraúba, Guará e Bem-te-vi. 


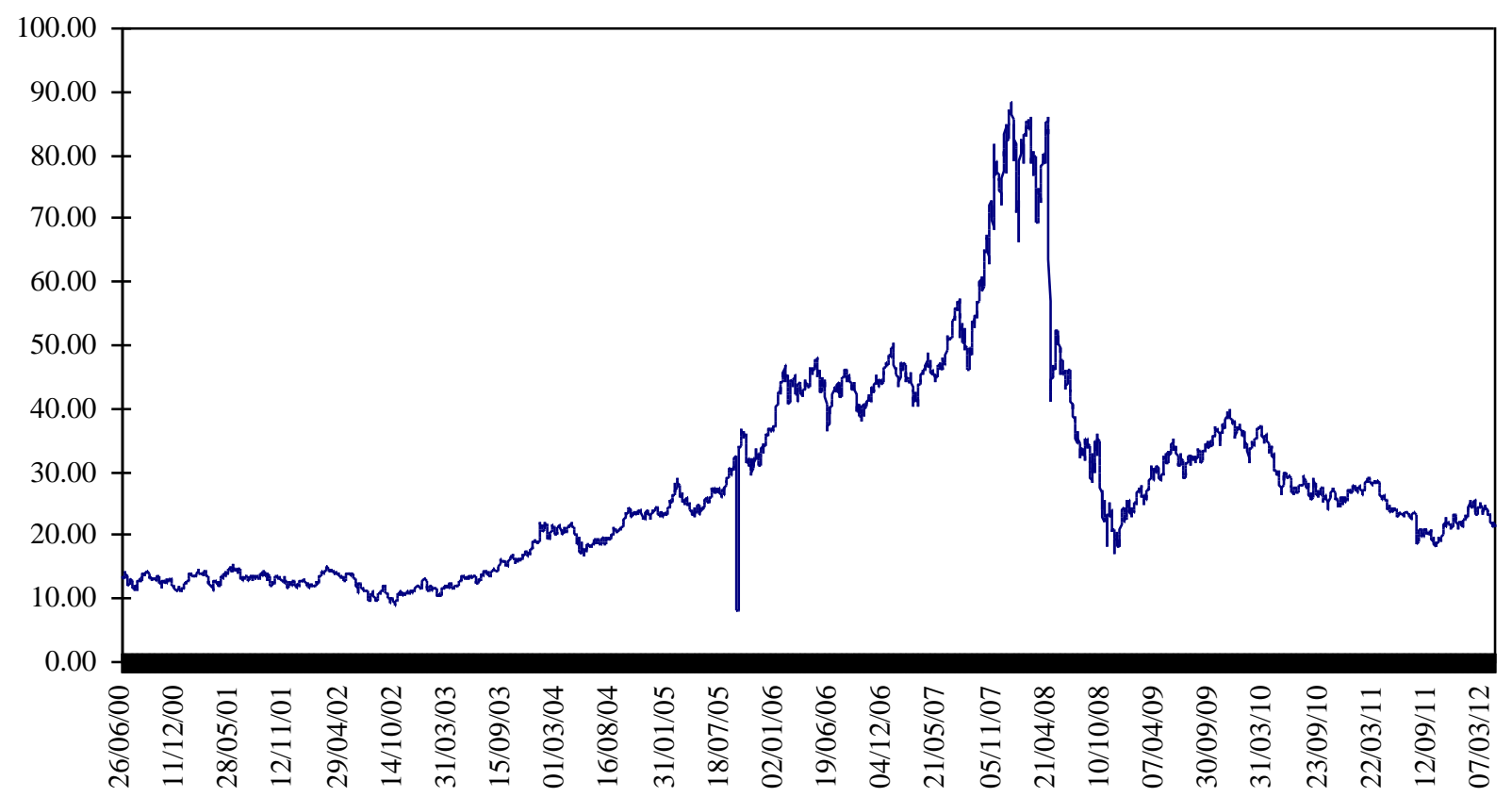

Gráfico 1: Evolução do preço (R\$) das ações da Petrobras - 26/06/2000 a 17/04/2012.

Fonte: elaboração própria a partir da base de dados.

Esses anúncios geraram uma variedade de notícias na imprensa que afetaram o mercado financeiro, bancos de investimento, empresas produtoras de petróleo e diversos agentes econômicos que fazem estimativas para o volume recuperável de petróleo e gás. Entretanto, a exploração dessas jazidas apresenta enormes desafios tecnológicos e de investimentos novos para a Petrobras. ${ }^{3}$

Observa-se que a trajetória de alta dos preços das ações da Petrobras ao longo de todo o período foi interrompida precisamente com a ocorrência da crise no mercado financeiro norte-americano em 2008. Desse ponto em diante, há uma breve recuperação e uma tendência de queda ao longo do tempo, refletindo certo pessimismo ou incerteza em relação aos lucros futuros da estatal brasileira.

\footnotetext{
${ }^{3}$ Ver Silva (2011).
} 


\subsection{Estatística descritiva da base de dados}

A rentabilidade diária foi calculada com base no preço de fechamento, em Reais correntes, de modo que o valor 0,02406, por exemplo, corresponde ao rendimento diário de 2,406\%. Isto é, para se obter a rentabilidade diária, empregou-se a seguinte expressão, a partir do preço de fechamento das ações:

$$
x=\frac{y_{t}-y_{t-1}}{y_{t}}
$$

Onde:

$x$ : rentabilidade diária.

$y_{t}$ : preço das ações, em $\mathrm{R} \$$

$y_{t-1}$ : preço das ações no dia anterior, em $\mathrm{R} \$$.

A tabela 1 apresenta as medidas descritivas da série de rentabilidade. A observação das medidas descritivas confirma a ideia de que a média, sendo muito menor que o desvio-padrão, não é uma boa medida para prever o comportamento futuro da rentabilidade das ações. O coeficiente de variação é 48,84615. Isto é, a dispersão em torno da média é altíssima. O coeficiente de assimetria indica que a maior parte das observações está situada abaixo da média da distribuição (assimetria positiva).

Tabela 1: Estatística descritiva da rentabilidade das ações

\begin{tabular}{c|c|c|c|c|c|c|c|c}
\hline \hline Min. & $1^{\circ}$ Quartil & Mediana & Média & $3^{\circ}$ Quartil & Max. & $\begin{array}{c}\text { Desvio- } \\
\text { Padrão }\end{array}$ & Assimetria & Curtose \\
\hline$-0,7517$ & $-0,01175$ & 0,0000 & 0,0013 & 0,0128 & 3,1380 & 0,0635 & 39,16524 & 1975,730 \\
\hline \hline
\end{tabular}

Fonte: elaboração própria.

Ademais, o coeficiente de curtose indica que a distribuição tem caudas bem mais pesadas que a distribuição gaussiana. Comportamento típico das variáveis financeiras. Outra informação que indica o alto grau de incerteza acerca do comportamento futuro do retorno das ações é a ausência de autocorrelação entre as observações. Isto pode ser constatado estimandose a função de autocorrelação dos retornos. 
O comportamento da função de autocorrelação entre as primeiras 100 observações aparece na figura 1 a seguir. A despeito de uma observação discrepante, todas as autocorrelações, nas diversas defasagens, podem ser consideradas estatisticamente nulas. Esta característica impede sua previsão a partir de modelos lineares auto-regressivos convencionais, mesmo para poucos passos à frente, pois a série de retornos não possui memória de curto prazo. O retorno atual não é influenciado pelo conjunto de informações e notícias do dia anterior.

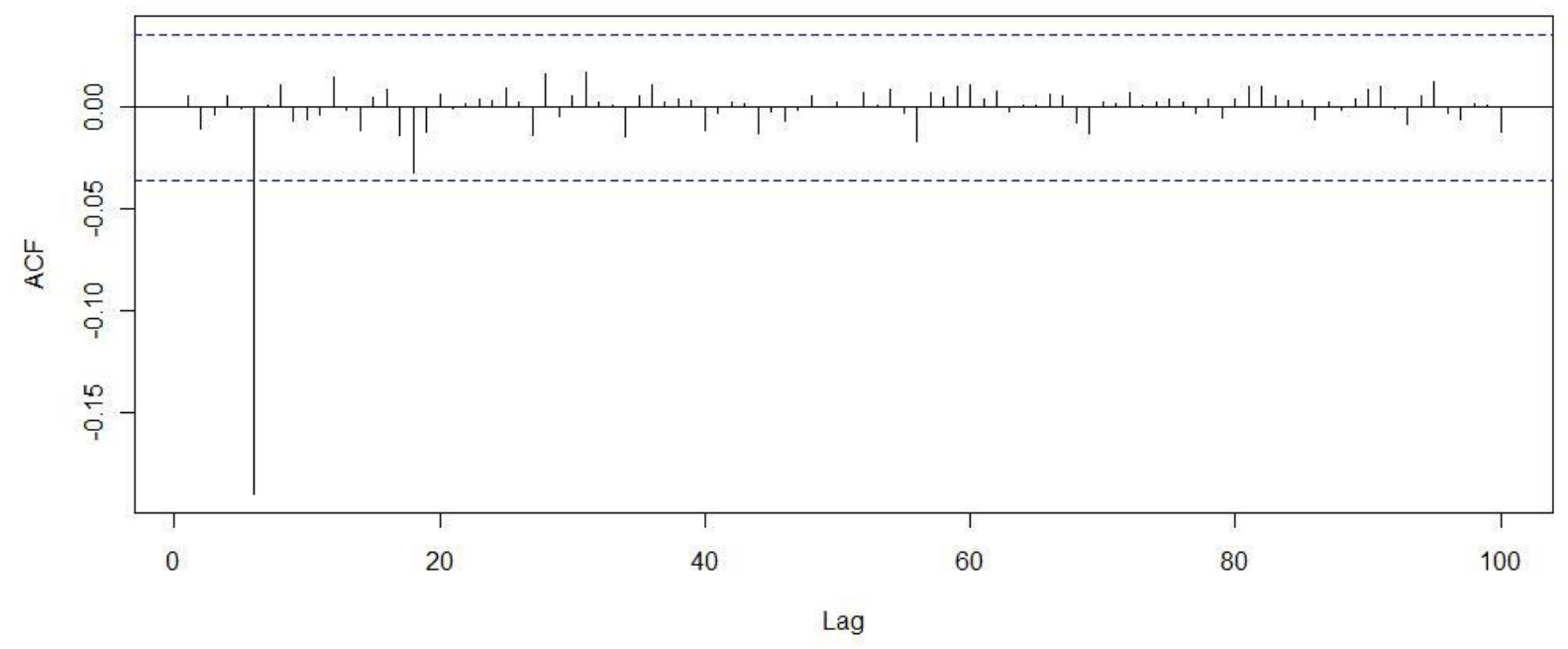

Figura 1: Função de autocorrelação do retorno das ações. Fonte: elaboração própria.

Portanto, obter as probabilidades associadas a cada rentabilidade pode ser uma estratégia mais eficiente para o investidor conhecer a rentabilidade futura das ações da Petrobras. O histograma de frequiência e as medidas descritivas acima podem dar uma idéia da f.d.p. teórica mais adequada para descrever o seu comportamento. 


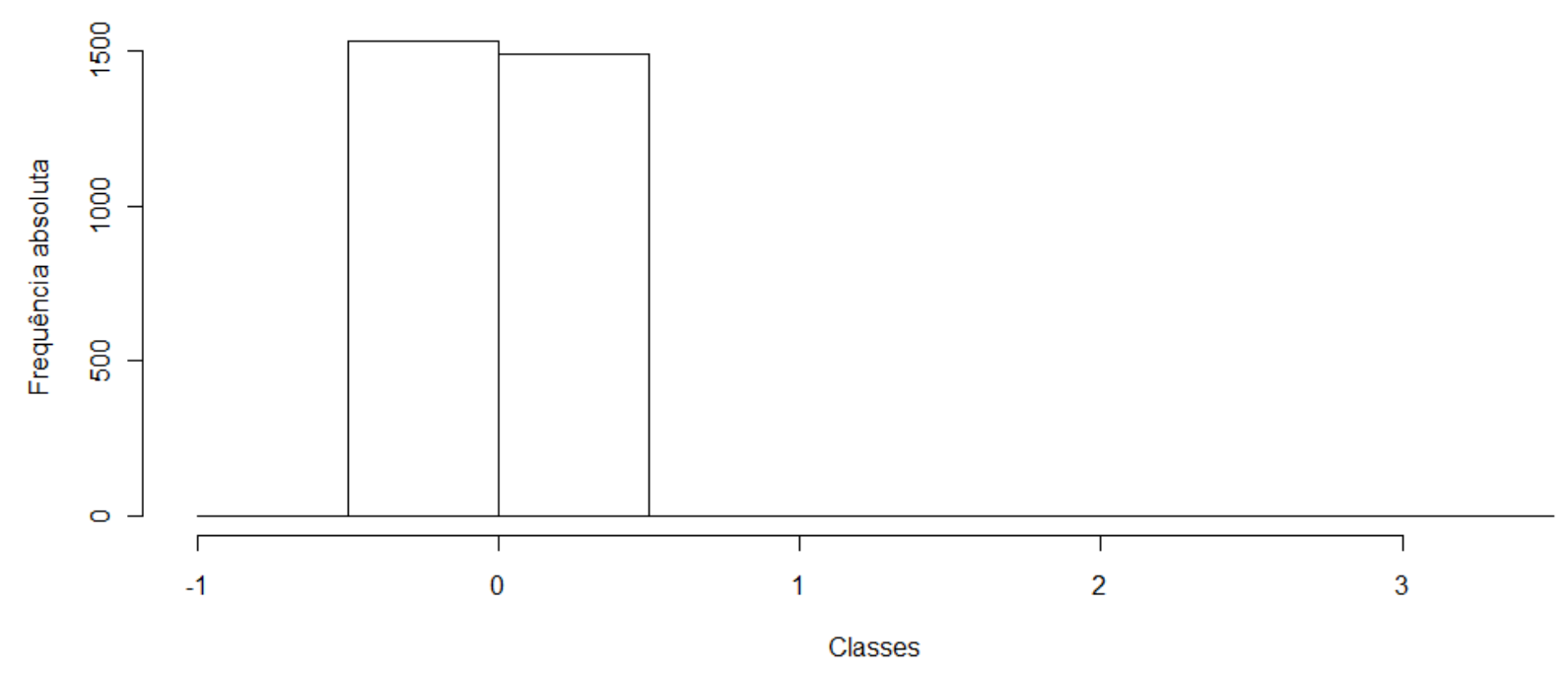

Figura 2: Histograma de freqüência da rentabilidade das ações Fonte: elaboração própria.

A tarefa seguinte é escolher densidades candidatas que possam descrever razoavelmente a rentabilidade das ações. À primeira vista, as medidas de assimetria e curtose apresentadas na tabela 1 sugerem a necessidade de uma densidade para variável contínua diferente da Normal, em especial com elevado grau de curtose (caudas pesadas).

\subsection{Escolha da função densidade de probabilidade}

As funções densidade de probabilidade consideradas candidatas mais prováveis para a rentabilidade do fundo de ações foram a Normal, a Logística e a Cauchy, uma vez que a variável rentabilidade é contínua e pode assumir tanto valores negativos quanto positivos.

Em vista de alterações nos parâmetros das densidades Normal, Logística e Cauchy, elas podem assumir formatos bastante específicos, mostrando grande versatilidade, podendo descrever uma variedade muito grande de fenômenos aleatórios. A função densidade de probabilidade Normal é definida pela expressão: 


$$
f(x)=\frac{1}{\sigma \sqrt{2 \pi}} \exp \left\{-\frac{1}{2}\left(\frac{x-\mu}{\sigma}\right)^{2}\right\}, \forall x .
$$

Com média $\mu$ e variância $\sigma^{2}$. A função densidade de probabilidade logística é definida pela expressão:

$$
f(x)=\frac{1}{s \exp \left[\frac{(x-m)}{s}\right]\left[1+\exp \left(\frac{(x-m)}{s}\right)\right]^{-2}}, \forall x .
$$

Onde a média corresponde ao parâmetro de localização $m, s$ é o parâmetro de escala e a variância é dada por $\frac{\pi^{2}}{3 s^{2}}$. A função densidade de probabilidade Cauchy é definida pela expressão:

$$
f(x)=\frac{l}{\pi s\left\{l+\left[\frac{(x-l)}{s}\right]^{2}\right\}}, \forall x .
$$

Em que $l$ é o parâmetro de localização e $s$ é parâmetro de escala da distribuição. Uma das principais diferenças entre a Normal, a Logística e a Cauchy é que esta última apresenta as caudas mais longas, possibilitando uma maior dispersão ao longo dos extremos da distribuição. Essa característica pode ser importante para captar a rentabilidade das ações. Para auxiliar nessa escolha, foram plotadas três densidades para $x \in(-30 ; 30)$.

No caso da Normal atribuiu-se o valor 2 para a média e 2,5 para o desvio-padrão. No caso da Cauchy e da Logística foram atribuídos os valores 2 e 2,5 para os parâmetros de localização e escala respectivamente. Desse modo, através dos gráficos das densidades mostrados abaixo foi facilitada a comparação com o comportamento da variável observada, mostrado na figura 2 acima.

À primeira vista, parece que as f.d.p Normal e Cauchy, para os parâmetros que foram atribuídos, são menos compatíveis do que a densidade Logística para o caso da rentabilidade das ações. Indicações mais precisas são dadas abaixo a partir da estimação dos parâmetros para a série diária de rentabilidade.

Uma indicação mais precisa acerca da função densidade que melhor descreve o comportamento da série de rentabilidade pode ser obtida pelo teste não paramétrico de Kolmogorov-Smirnov, cuja estatística de teste é obtida através da expressão: 


$$
D_{n}=\sup _{1 \leq i \leq n}\left|F\left(x_{i}\right)-F_{n}\left(x_{i}\right)\right|,
$$

que é o limite superior extremo das diferenças entre os valores absolutos da distribuição acumulada empírica e teórica consideradas no teste. A hipótese nula é rejeitada se o valor de $D_{n}$ for maior que o valor tabelado, ou, de forma equivalente, se a probabilidade exata do teste for menor que o nível de significância.

Uma vez que tenha sido detectada a função densidade de probabilidade que melhor descreve o comportamento da rentabilidade do fundo de ações, com o emprego da função de distribuição acumulada $(F(x))$, podem ser calculadas as probabilidades associadas ao valor da rentabilidade para alguns períodos futuros. Desse modo, pode-se calcular a probabilidade de um valor desejado $X$ ser maior que um valor observado $x$ :

$$
P[X \geq x]=1-F(x)=1-\int_{-\infty}^{x} f(x ; \boldsymbol{\theta}) d x,
$$

em que $f(x ; \boldsymbol{\theta})$ é a função densidade de probabilidade que descreve o comportamento de $X$.

(a)

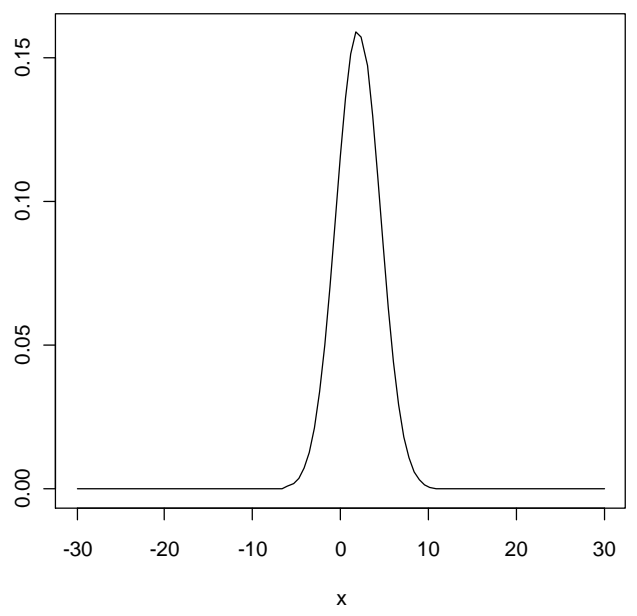


(b)

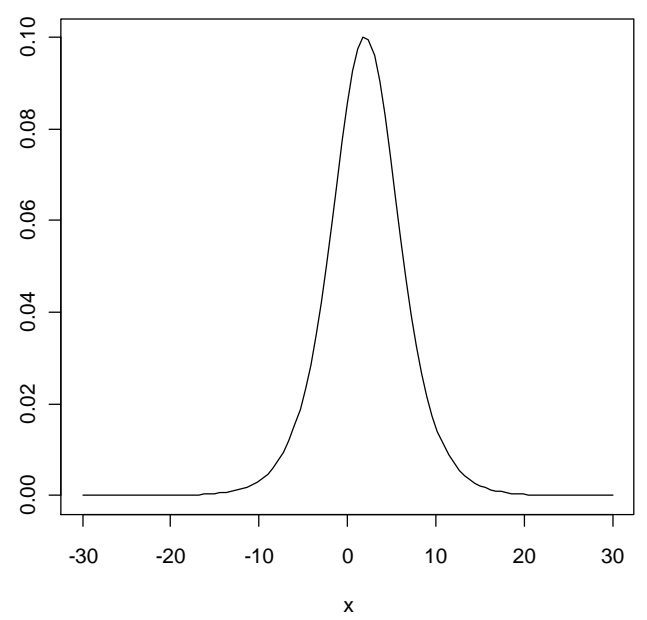

(c)

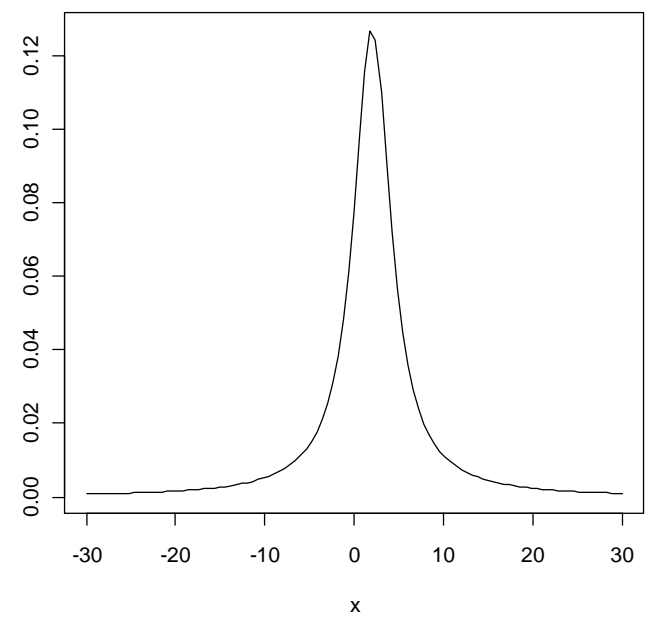

Figura 3: - (a) Normal - (b) Logística - (c) Cauchy Fonte: elaboração própria.

Como a rentabilidade é registrada diariamente, o investidor poderá também estar interessado na rentabilidade mensal ou até anual, para isso basta fazer a conversão pela equivalência das taxas.

\section{RESULTADOS E DISCUSSÃO}

\subsection{Estimativas dos parâmetros}

A estimativa dos parâmetros considerando as três funções densidade candidatas, a partir da série de rentabilidade, também foi efetuada no Ambiente $\boldsymbol{R}$. Há pelo menos três alternativas bem conhecidas para se estimar os parâmetros de uma função densidade de probabilidade nesse software: o método analógico, o método dos momentos e o método da máxima verossimilhança. Adotou-se este último procedimento, em vista das propriedades assintóticas altamente desejáveis do estimador de máxima verossimilhança ${ }^{4}$. Os resultados da estimativa são apresentados na tabela 2, abaixo.

\footnotetext{
${ }^{4}$ Ver Greene (2006: Cap. 17) e Ramanathan (1993).
} 
Tabela 2: Estimativa dos parâmetros das densidades normal e logística para a rentabilidade

\begin{tabular}{c|c|c|c|c}
\hline \hline Parâmetros & $n$ & Normal & Logística & Cauchy \\
\hline Média/localização & \multirow{2}{*}{3028} & $\begin{array}{c}0,001265792 \\
(0,0011531758)\end{array}$ & $\begin{array}{c}0,0006694661 \\
(0,0003926774)\end{array}$ & $\begin{array}{c}0,0008171474 \\
(0,0003051285)\end{array}$ \\
\hline $\begin{array}{c}\text { Desvio- } \\
\text { padrão/escala }\end{array}$ & \multirow{2}{*}{3028} & $\begin{array}{c}0,063456111 \\
(0,0008154184)\end{array}$ & $\begin{array}{c}0,0128446568 \\
(0,0001951018)\end{array}$ & $\begin{array}{c}0,0110374141 \\
(0,0002632915)\end{array}$ \\
\hline$L(x, \hat{\boldsymbol{\theta}})$ & & 4052,882 & 6992,68 & 7049,31 \\
\hline \hline
\end{tabular}

Nota: entre parênteses os erros padronizados (desvio-padrão dos resíduos) extraídos da matriz de informação, calculada por aproximação numérica.

Fonte: elaboração própria.

Os resultados expostos na Tabela 2 indicam, à primeira vista, que as densidades Cauchy e Logística, de caudas mais pesadas que a Normal, são fortes candidatas para descrever o comportamento da rentabilidade das ações da Petrobras, pois o valor da função de máxima verossimilhança é sensivelmente maior que no caso da Normal em ambos os casos. Com relação aos erros-padrão dos coeficientes estimados, que são um índice da eficiência do estimador, nota-se, que no caso da Normal, eles são sensivelmente maiores que os das demais densidades. Porém, neste aspecto, a Logística e a Cauchy são muito similares, com uma pequena diferença aparentemente favorável à densidade Cauchy.

Com relação à escolha entre as densidades, para grandes amostras, o teste QuiQuadrado e o teste Kolmogorov-Smirnov têm o mesmo poder. A hipótese nula do teste é de que a variável foi extraída de uma população específica, postulada pelo pesquisador. No presente caso as densidades a serem consideradas no teste serão a Normal, a Logística e Cauchy. O critério de escolha da densidade mais representativa para a rentabilidade foi o maior valor da probabilidade exata do teste, que é um procedimento padrão em estudos dessa natureza e que corresponde ao menor valor calculado da estatística $D_{n}$.

Com os procedimentos acima descritos, os resultados do teste de hipóteses de Kolmogorov-Smirnov para a hipótese nula para a densidade de interesse são apresentados na tabela 3 . 
Tabela 3: Resultados do teste de Kolmogorov-Smirnov

\begin{tabular}{c|c|c}
\hline \hline Hipótese nula & $\mathrm{D}_{\mathrm{n}}$ & Probabilidade exata \\
\hline Densidade Normal & 0,2642 & 0,0000 \\
\hline Densidade Logística & 0,0459 & 0,0034 \\
\hline Densidade Cauchy & 0,0578 & 0,0000 \\
\hline
\end{tabular}

Fonte: elaboração própria.

Observando-se os resultados do teste de Kolmogorov-Smirnov contata-se que a probabilidade exata do teste, para o caso da densidade Logística, é muito superior à das densidades Normal e Cauchy, que possuem também um valor sensivelmente maior para a estatística $D_{n}$, sugerindo a rejeição da hipótese nula da Normal e da Cauchy.

Com esse resultado pode-se inferir que a densidade de probabilidade que melhor descreve o comportamento da rentabilidade diária das ações PTR4 da Petrobras é a densidade Logística. A 0,34\% de significância não se pode rejeitar a hipótese nula de que a rentabilidade do fundo de ações é descrita por uma densidade Logística com os parâmetros apresentados na tabela 2.

O cálculo das probabilidades de rentabilidade diária foi feito com base na Função de Distribuição Acumulada Logística, definida pela expressão (5), com os parâmetros estimados, expostos na tabela 2. Os resultados do cálculo das probabilidades associadas à rentabilidade do fundo são apresentados na tabela 4. A taxa de retorno equivalente (acumulada) mensal, para o mês de 22 dias úteis, aparece dentro dos parênteses. 
Tabela 4: Estimativa das probabilidades de rentabilidade das ações

\begin{tabular}{|c|c|c|}
\hline $\begin{array}{c}\text { Rentabilidade diária }(X) \\
\qquad p[X \geq x]\end{array}$ & $\begin{array}{l}\text { Medida } \\
(x)\end{array}$ & Probabilidade \\
\hline$P[X \geq 0,000000]$ & Mediana & 0,5130271 \\
\hline$P[X \geq-0,01175]$ & $1^{\circ}$ Quartil & 0,7245007 \\
\hline$P[X \geq 0,0006694661](1,48 \% \text { ao mês })^{*}$ & Localização & 0,5000000 \\
\hline$P[X \geq 0,00130]$ (2,9\% ao mês $)$ & Média amostral & 0,4877302 \\
\hline$P[X \geq 0,06476659]$ & Média +1 desvio & 0,0067585 \\
\hline$P[X \geq 0,01351412]$ & Localização + 1 escala & 0,2689414 \\
\hline$P[X \geq 0,01280000](32,29 \%$ ao mês $)$ & $3^{\circ}$ Quartil & 0,2800118 \\
\hline$P[X \geq 0,0006769842]$ (1,5\% ao mês) & --------------- & 0,4998537 \\
\hline$P[X \geq 0,001000](2,2 \%$ ao mês $)$ & --------------- & 0,4935671 \\
\hline$P[X \geq 0,001344485]$ (3\% ao mês) & --------------- & 0,4868649 \\
\hline$P[X \geq 0,001564924]$ (3,5\% ao mês) & --------------- & 0,4825784 \\
\hline$P[X \geq 0,00178435]$ (4\% ao mês) & --------------- & 0,4783142 \\
\hline$P[X \geq 0,002002770]$ (4,5\% ao mês) & --------------- & 0,4740727 \\
\hline$P[X \geq 0,002220196]$ (5\% ao mês) & ------------- & 0,4698542 \\
\hline
\end{tabular}

*Considerando-se um mês com 22 dias úteis.

Fonte: cálculos do autor.

A interpretação desses resultados deve ser feita do seguinte modo. Se o investidor estiver interessado em saber qual a probabilidade de se obter uma rentabilidade diária maior do de 0,0006769842 ao dia (maior do que 1,5\% ao mês), deve esperar esse resultado com uma probabilidade de 0,4998537 .

A rentabilidade diária será maior do que a mediana com uma probabilidade de 0,5130271. Isto é, em mais de 50\% dos casos a rentabilidade diária será positiva. Quando se considera a rentabilidade acumulada para o mês de 22 dias úteis, outros resultados emergem. Observa-se que a probabilidade decai à medida que o retorno das ações se eleva. Ainda assim, a probabilidade de se obter uma rentabilidade maior do que $5 \%$ ao mês no longo prazo é bastante elevada (0,4698542). Esses resultados podem ajudar, em parte, a explicar a alta liquidez das ações da Petrobras, cujo índice é sensivelmente superior ao das demais ações de empresas de petróleo negociadas na BOVESPA (ver tabela 1).

Uma forma de visualizar mais claramente a combinação rentabilidade (abscissa) versus probabilidade (ordenada) aparece no gráfico a seguir, que expressa os pares ordenados com uma tendência linear, a partir dos resultados da tabela 4. 


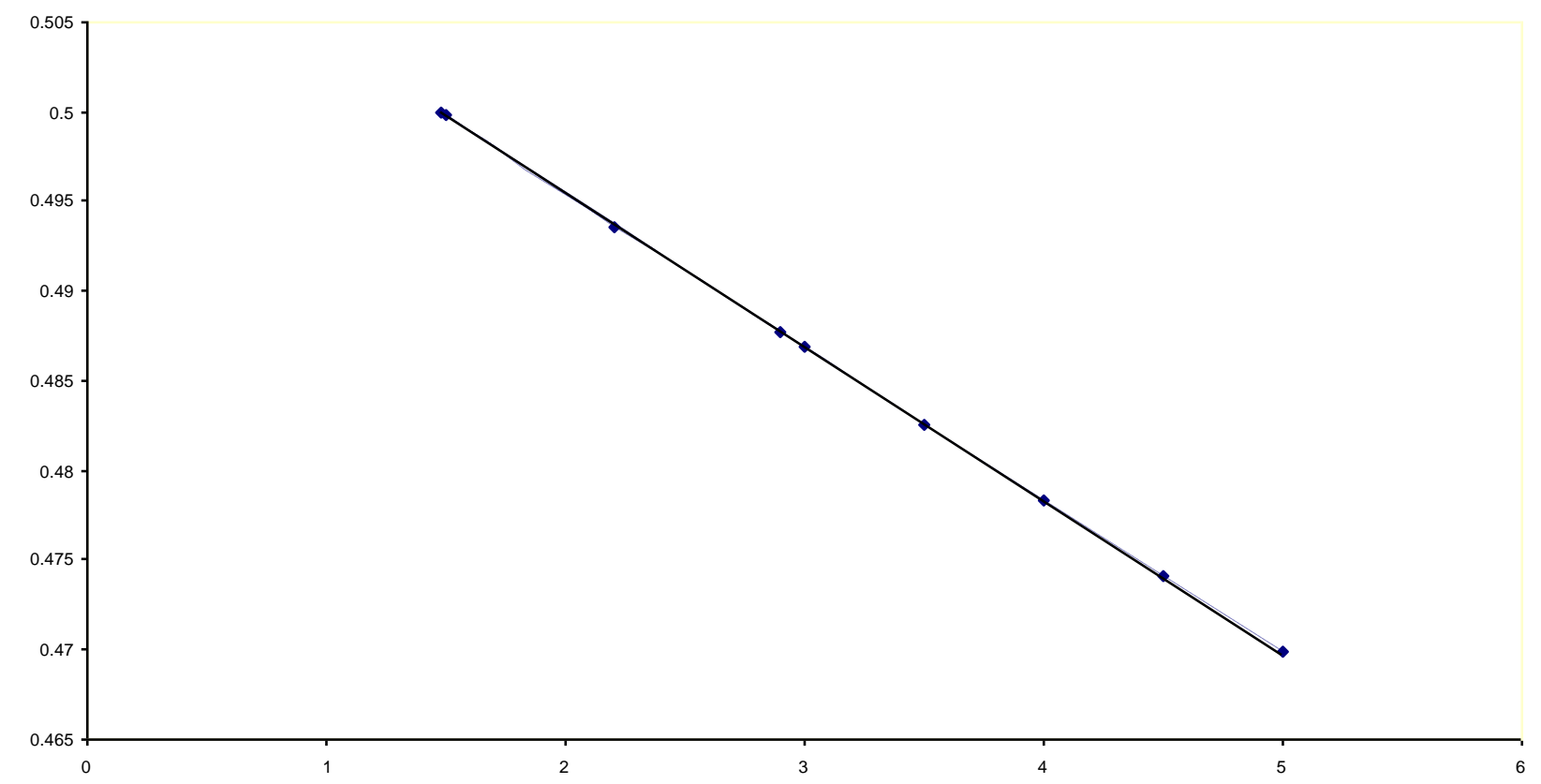

Gráfico 2: Rentabilidade versus probabilidade das ações da Petrobras. Fonte: elaboração própria.

Com as novas descobertas de petróleo no Brasil, nos últimos quatro anos, é de esperar que, à medida que as expectativas iniciais sobre o volume recuperável de óleo e gás natural forem se confirmando através de novos testes e perfurações, a liquidez e o retorno das ações persistam no longo prazo, assim como sua probabilidade de ocorrência. Todavia, os desafios tecnológicos e a necessidade de novos investimentos para a Petrobras, a partir das características geológicas das novas descobertas, são consideráveis e impõem a necessidade de novas emissões primárias, a baixo custo para a empresa, como fonte de financiamento da acumulação de capital fixo e aquisição de novas tecnologias.

\section{CONCLUSÃO}

O objetivo do trabalho foi testar três densidades de probabilidade para prever o comportamento da rentabilidade diária das ações preferenciais da Petrobras (PETR4), a partir de seu preço diário de fechamento na BOVESPA para os dias úteis no período de 26/06/2000 
a 17/04/2012. A partir das medidas descritivas do retorno das ações e das características da variável, foram selecionadas três densidades candidatas.

Com a estimação dos parâmetros das três densidades e com a aplicação do teste nãoparamétrico de Kolmogorov-Smirnov, constatou-se que a função que melhor descreve a rentabilidade do fundo de ações é a densidade Logística. Com esse resultado, a partir da Função de Distribuição Acumulada, foi possível estimar as probabilidades de rentabilidade diária e sua equivalente mensal.

A estimativa indica que a probabilidade de se obter rentabilidade diária positiva é de 0,5130271. Isto é, em mais da metade dos dias úteis do mês o investidor deverá obter rentabilidade positiva. Se o investidor deseja uma rentabilidade maior do que 3\% ao mês, pode esperar obtê-la com uma probabilidade de 0,4868649. Num horizonte de longo prazo, a melhor estimativa de rentabilidade diária será de 0,0006694661 ao dia, o que equivale a uma rentabilidade esperada de $1,48 \%$ ao mês.

Um trabalho futuro poderá averiguar outras possibilidades, por exemplo, incluindo novas densidades candidatas que possam melhor caracterizar o comportamento do retorno das ações, como a densidade hiperbólica generalizada ${ }^{5}$, que não foi objeto de estudo dentro dos limites do trabalho.

\section{REFERÊNCIAS BIBLIOGRÁFICAS}

CARGNELUTTI FILHO, A.; MATZENAUER, R.; TRINDADE, J. K. (2004) “Ajustes de funções de distribuição de probabilidade à radiação solar global no Estado do Rio Grande do Sul”, Pesquisa Agropecuária Brasileira, Brasília, 39(12): 1157-1166.

CATALUNHA, M. J.; SEDIYAMA, G. C.; LEAL, B. G.; SOARES, C. P. B.; RIBEIRO, A. (2002) "Aplicação de cinco funções densidade de probabilidade a séries de precipitação pluvial no Estado de Minas Gerais", Revista Brasileira de Agrometeorologia, Santa Maria, 10(1): 153-162.

DE NEGRI, J. A. et. al. (Org.) (2010) Poder de Compra da Petrobras: impactos econômicos nos seus fornecedores. Brasília: IPEA: Petrobras.

\footnotetext{
${ }^{5}$ Ver Hu (2005).
} 
GRAZIANI, A. (2003) The Monetary Theory of Production, Cambridge: Cambridge University Press.

GREENE, W. (2006) Econometric Analysis. London, UK: Pearson Education.

HU, W. (2005) "Calibration of multivariate generalized hyperbolic distributions using EM algorithm, with applications in risk management, portfolio optimization and portfolio credit risk", Electronic Theses, Treatises and Dissertations. Paper 3694, Florida State University.

BOVESPA (2012) Índice Ibovespa: definição e metodologia. São Paulo: IBOVESPA. Disponível em: www.bmfbovespa.com.br/Pdf/Indices/IBovespa.pdf ; Acesso 02.05.2012.

LABINI, P. S. (1986) Oligopólio e Progresso Técnico. São Paulo: Nova Cultural.

MORETTIN, L. G. (1986) Estatística Básica: probabilidade. São Paulo: Livraria Ciência e Tecnologia Editora Ltda.

MURTA, R. M.; TEODORO, S. M.; BONOMO, P.; CHAVES, M. A. (2005) "Precipitação Pluvial Mensal em níveis de probabilidade pela distribuição Gama para duas localidades do sudoeste da Bahia", Ciência e Agrotecnologia, Lavras, vol. 29(5): 988-994.

PETERNELli, L. A.; MELlO, M. P. (2007) Conhecendo o R: uma visão estatística. Viçosa: Universidade Federal de Viçosa.

RAMANATHAN, R. (1993) Statistical Methods in Econometrics. London: Academic Press.

R DEVELOPMENT CORE TEAM (2007). "R: A language and environment for statistical computing. R Foundation for Statistical Computing", Vienna, Austria. URL < http://www.Rproject.org>.

SILVA, F. R. C. (2011) Descobertas de petróleo e crescimento econômico no Brasil: simulações a partir do modelo de Solow. Natal, RN: UFRN (Monografia de Graduação).

STEINDL, J. (1983) Maturidade e estagnação no capitalismo americano. São Paulo: Abril Cultural. 